\title{
Novel Diindolylmethane derivatives based NLC formulations to improve the oral Bioavailability and anticancer effects in Triple Negative Breast Cancer
}

Chandraiah Godugu ${ }^{1,2,{ }^{*},}$ Ravi Doddapaneni ${ }^{*}$, Stephen H Safe ${ }^{4}$ and Mandip Singh ${ }^{1 \#}$

${ }^{1}$ College of Pharmacy and Pharmaceutical Sciences, Florida A\&M University, Tallahassee, Florida, USA

${ }^{2}$ Department of Regulatory Toxicology, National Institute of Pharmaceutical Education and Research, (NIPER), Hyderabad, Telangana, India-500037

${ }^{3}$ The Daniel K. Inouye College of Pharmacy, University of Hawaii at Hilo, Hilo, HI, USA

${ }^{4}$ Department of Veterinary Physiology and Pharmacology, Texas A\&M University, College Station, TX, USA

* Both the authors contributed equally

"Corresponding Author: Mandip Singh; Phone: (850) 561-2790; Fax: (850) 599-3813;

E-mail: mandip.sachdeva@gmail.com 


\section{Abstract}

The present study demonstrates the promising anticancer effects of novel C- substituted diindolylmethane (DIM) derivatives DIM-10 and DIM-14 in aggressive TNBC models. In vitro studies demonstrated that these compounds possess strong anticancer effects. Caco-2 permeability studies resulted in poor permeability and poor oral bioavailability was demonstrated by pharmacokinetic studies. Nano structured lipid carriers (NLC) formulations were prepared to increase the clinical acceptance of these compounds. Significant increase in oral bioavailability was observed with NLC formulations. Compared to DIM-10, DIM-10 NLC formulation showed increase in $\mathrm{C}_{\max }$ and $\mathrm{AUC}$ values by 4.73 and 11.19 -folds, respectively. Similar pattern of increase was observed with DIM-14 NLC formulations. In dogs DIM-10 NLC formulations showed an increase of 2.65 and 2.94-fold in $\mathrm{C}_{\max }$ and AUC, respectively. The anticancer studies in MDA-MB-231 orthotopic TNBC models demonstrated significant reduction in tumor volumes in DIM-10 and DIM-14 NLC treated animals. Our studies suggest that NLC formulation of both DIM-10 and 14 are effective in TNBC models.

Key words: Triple Negative breast cancer (TNBC), Diindolylmethane, Nano structured lipid carriers, Oral Bioavailability 


\section{Introduction}

Triple negative breast cancer (TNBC) is defined by tumors that do not express estrogen, progesterone and human epidermal growth factor receptors, representing approximately $20 \%$ of breast cancers. These types of cancers are clinically more aggressive. Currently available chemotherapy based treatment options for TNBC exhibit poor therapeutic benefits and possess serious toxicity issues (O'Toole, Beith et al. 2013, Jamdade, Sethi et al. 2015). Considering the challenges involved in treating TNBC and its inherent poor prognosis, there is a need for novel and safer drugs to treat this type of cancer. Recent research is focused on the use of natural products to combat various types of cancer (Godugu, Patel et al. 2014, Siddiqui, Singh et al. 2015). Natural products are available in various dietary supplements; therefore, consumption of these compounds at early stages of cancer may act as chemopreventive agents. On the other hand, the oral route, which is most desirable in anticancer therapy is not possible with many anticancer natural product drugs due to their inherent water insolubility and poor oral absorption. One of the classical examples is curcumin, which has demonstrated to have promising anticancer effects in various cancer types, however, its poor aqueous solubility and limited oral bioavailability limited its broad spectrum applications. Bis(31-indolyl)methane (or 3, $3^{1}$ diindolylmethane (DIM) is an active metabolite of indole-3-carbinol derived from cruciferous vegetables and exhibits a broad spectrum of anticancer activities. DIM compounds have been reported to be useful in combating various cancer types (York, Abdelrahim et al. 2007, Andey, Patel et al. 2013). However, DIM has poor oral bioavailability due to its low solubility/high lipophilicity and has limited oral absorption (Jellinck, Makin et al. 1993). Pharmacokinetic studies with DIM indicated that dose-dependent absorption and nonlinear increase in plasma maximum concentrations $\left(\mathrm{C}_{\max }\right)$, suggesting the saturation of systemic absorption (Reed, Arneson et al. 2006, Reed, Sunega et al. 2008). In another study, microencapsulated extended release DIM formulations produced increased oral bioavailability, suggesting that solubility is the rate-limiting 
step in oral bioavailability (Reed, Sunega et al. 2008). Although few studies are available on unsubstituted DIM based anticancer effects, their potency were found to be low and C-substituted DIM-analogues were reported to be superior in producing the anticancer effects in lung, breast, prostate cancer etc. Recently we have also demonstrated that novel self emulsified based DIM14 and DIM-P formulations produce better anticancer effects in lung cancer models (Ref). Therefore, development of novel orally active C-substituted DIM derivatives (C-DIM analogs) will have major clinical implications for the treatment of TNBC. Among different types of C-DIM analogs reported, DIM-10 and DIM-14 are expected to have significant impact in the treatment of TNBC.

The supplementary figure 1 shows the chemical structure of DIM-10 and DIM-14. Our vitro anticancer studies suggested that both DIM-10 and DIM-14 have promising anticancer effects on TNBC cells. However, these compounds found to possess poor aqueous solubility. The Caco-2 permeability studies also indicated the poor permeability. To overcome the limited solubility and associated poor oral bioavailability problems of these promising C-DIM analogs, novel nanotechnology based oral formulations were attempted. There is a growing interest in enhancing the bioavailability of poorly bioavailable drugs using novel nanoformulations mediated enhanced lymphatic absorption. In order to bring these compounds more closer to the human applications as orally administrable anticancer and chemopreventive agents, we have developed DIM-10 and DIM-14 loaded nano structured lipid carriers (NLCs) based novel nanoformulations (Beloqui, Solinis et al. 2013). The pharmacokinetic (PK) studies were performed in rats and selected formulations were further studied in dogs. Finally, these formulations were evaluated in MDA-MB-231 orthotopic TNBC mouse models. This is the first attempt to prepare DIM-10 and DIM-14 loaded NLC formulations and demonstrate their anticancer effects in orthotopic TNBC nude mode models. 


\section{Materials and Methods}

2.1. Materials: DIM-10 and DIM-14 were synthesized and provided by Dr Stephen Safe (Texas A\&M University). The primary antibodies Cleaved Caspase 3, Cyclin D1, PCNA and B-actin were procured from Santa Cruz Biotechnology Inc. Protein assay kit was purchased from Pierce $^{\mathrm{TM}}$ BCA Protein Assay Kit. VEGF kit was purchased from Thermo Fisher scientific Inc. Human TNBC cell lines (MDA-MB-231, 453 and 468) and other breast cancer cell lines such as SKBR3 and BT474 cells and Caco-2 cell lines were procured from ATCC, USA.

2.2. DIM-10 and DIM-14 in vitro anticancer activities: In vitro cytotoxicity, cell migration and clonogenic studies were performed to demonstrate the anticancer activity of DIM-10 and -14 on TNBC cell lines (MDA-MB-231, MDA-MB-468 and MDA-MB-435 cells). For cytotoxicity assay, TNBC cells were plated in 96 well plates at 10,000 cells/well density. After overnight incubation, cells were treated with various concentrations of DIM-10 and DIM-14, $0.1 \%$ DMSO treated cells were considered as control cells. After $72 \mathrm{~h}$ treatment, cells were fixed and cell viability was measured by crystal violet staining $(0.05 \%)$. Cell viability Percentage was calculated by considering DMSO treated control cells as $100 \%$ viable. Clonogenic assay was performed to demonstrate the inhibitory effects C-DIMs on cell reproduction. For this assay, MDA-MB-468 cells were placed in 24 - well plate at a density of 100 cells/well, after $24 \mathrm{~h}$ cells were treated with DIM-10 and DIM-14. The cells were washed with media 48h after the treatment and were allowed to grow for 2 weeks, during this period medium were changed every alternative day. The individual cells grown as spheroids were fixed with glutaraldehyde and stained with crystal violet. The groups of cells more than 50 in number were considered as colonies. Colonies were counted under microscope, their sizes were recorded, and representative spheroid images were captured. 
Effect of DIM-10 and DIM-14 on cell migration: Cell migration assay is performed to study the antimetastatic effect of compounds in in-vitro conditions. In this assay 50,000/well of MDA-MB231 cells were placed in 24 well plates, cells were allowed to grow near to full confluence, after reaching the confluence, after which in the middle portion of each well, cells were scratched by using pipette tip, which result in the gap (injury formation). Scratched cells were removed by washing with PBS. Then cells were treated with different concentrations of DIM-10 and DIM-14. After $48 \mathrm{~h}$ the width of the gaps were measured microscopically and images were captured. In control cells due to high migratory potential of MDA-MB-231 cells, move into scratched gaps, which will result in narrowing down (closure) of gaps. The width of the gap was measured in all wells and effect of DIM-10 and -14 on cell migration was calculated.

2.3. Experimental solubility and lipophilicity: Saturation solubility measurements were performed using shake-flask method followed by reverse-phase HPLC analysis. The details of this studies was provided in supplementary information.

2.4. Preparation of NLC formulations: NLC formulations were prepared with optimized proportions of the following ingredients: Drug DIM-10 or 14, Compritol 888, Myglyol 812, Vitamin E TPGS and water was used as vehicle. All the above-mentioned formulation excipients are FDA approved and found to be safe in clinical applications. C-DIMs loaded NLC formulations were prepared by hot melt homogenization technique (Patlolla, Chougule et al. 2010). In brief, $20 \mathrm{mg}$ of drug was dissolved in dichloromethane and mixed with lipid phase comprised of Compritol $(7.0 \% \mathrm{w} / \mathrm{w})$ and Miglyol $(3.0 \% \mathrm{w} / \mathrm{w})$. Later, organic phase was removed on a rotary evaporator for $2-3 \mathrm{~h}$ at $80^{\circ} \mathrm{C}$ and to the heated lipid phase the aqueous solution (40 $\mathrm{ml}$ ) containing vitamin E TPGS $(1.5 \% \mathrm{w} / \mathrm{w})$ surfactant was added at same temperature under high speed mixing using a polytron homogenizer at 20,000 rpm for 1-2 min. The resultant oil-in-water dispersion was passed through nano-DeBEE high-pressure homogenizer at 5000 psi for 5 cycles. Throughout the process temperature was maintained at $80^{\circ} \mathrm{C}$. Prepared formulation was 
cooled to room temperature while stirring. This process resulted in the hardening of NLCs with drug entrapped in it. Prepared nanoparticles were analyzed for size and surface charge using BI-90 particle sizer (Brookhaven Instruments, Boston, USA), which is based on the principle of dynamic light scattering and Zeta potential measurement was carried with Zeta plus (Brookhaven Instruments). The entrapment efficiency of drug in NLC was measured according to the standard protocols.

2.5. In vitro drug release studies: In vitro drug release profile of DIM-10 and DIM-14 NLCs were studied in PBS containing tween 80 at $\mathrm{pH}$ 5.8. The details were given in supplementary information.

2.6. Caco-2 permeability studies: For in vitro permeability study Caco-2 cells were maintained and permeability studies were performed as per our reported method (Godugu, Patel et al. 2014). The detail of this study was provided in supplementary information.

2.7. Animals: Male Sprague Dawley rats (320-350g weights) were used for pharmacokinetic studies. Female nude mice (5-6 weeks age) were used for in vivo tumor studies. IACUC of FAMU approved all the animal protocols and during the experiments, animals were handled humanly. The details of the study are provided in supplementary information.

2.8. Pharmacokinetic studies: Initially, the oral bioavailability profile of both DIM-10 and DIM14 free drugs were evaluated in Sprague Dawley rats. The oral PK results indicated that both these promising C-DIMs have limited oral absorption. To overcome this limitation, we prepared NLC formulations, the oral bioavailability and Pharmacokinetic profile of DIM-10-NLC and DIM14-NLC formulations was studied. DIM-10 free drug at the dose of 30 and $100 \mathrm{mg} / \mathrm{kg}$ and DIM$10-\mathrm{NLC}$ at the dose of $30 \mathrm{mg} / \mathrm{kg}$ was administered orally. In case of DIM-14, $30 \mathrm{mg} / \mathrm{kg}$ oral dose of free drug and NLC formulation was administered. Rats were fasted overnight before the start of the experiments and randomly divided into different experimental groups. After oral 
administration of these drugs as free drugs and NLC formulations, blood samples $(250 \mu \mathrm{l})$ were withdrawn from tail veins at predetermined time points $(0.25,0.5,1,2,4,6,8,12,18$ and $24 \mathrm{~h}$ and rest of the procedure for pharmacokinetic study is followed as per our previous methods.

2.9. PK study in dogs: Pharmacokinetic profile of DIM-10 and its NLC formulation was studied in dogs after intravenous and oral administration at a dose of $5 \mathrm{mg} / \mathrm{kg}$. Animals were randomly distributed into three experimental groups $(n=3-4)$. A central venous catheter on long saphenous vein was placed on each dog on the day of study. Overnight fasted dogs were used for the PK study. Blood samples were collected from the venous catheters into heparinized tubes. Samples were collected at $0,0.25,0.5,1,2,4,6,8,12,18$ and $24 \mathrm{~h}$ after administration of a single dose of DIM-10 solution and NLC formulation.

2.10. Anticancer studies in orthotopic TNBC mouse models: MDA-MB-231 cells induced orthotopic breast cancer model was used for anticancer evaluations of DIM formulations. MDAMB-231 cells (2 million in $100 \mu$ PBS) were injected orthotopically into mammary fat pads. DIM10 and DIM-14, free drugs and NLC formulations were administered at the dose of $30 \mathrm{mg} / \mathrm{kg}$ daily orally for 4 weeks and tumor sizes were monitored every week. Four weeks after drug treatment animals were sacrificed; final tumor volumes and weights were recorded. Tumor sizes and volumes analysis was done as per standard methods (Supplementary information).

2.11. Western blot analysis: The protein extraction, sample preparation, SDS page and transfer of proteins to nitrocellulose membrane was performed according to our previous methods (Godugu, Patel et al. 2013). Membranes were probed with antibodies against cleaved caspase 3 (1:1000), Cyclin D1 (1:1000), PCNA (1:1000), and $\beta$-actin, all these primary antibodies were purchased from Santa Cruz Biotechnology, Santa Cruz, CA. Horseradish peroxidase-conjugated secondary antibodies (Santa Cruz Biotechnology, Santa Cruz, CA) were used. Proteins were visualized using enhanced chemiluminescent solution (Pierce, Rockford, 
IL) and exposed to Kodak X-OMAT AR autoradiography film (Eastman Kodak, Rochester, NY). The intensity of the western blot bands was quantified by densitometric analysis using imageJ software.

2.12. Quantification of VEGF by ELISA: VEGF levels were estimated in tumor homogenates by ELISA method (Godugu, Patel et al. 2013). Tumors were homogenized in RIPA lysis buffer containing protease inhibitors. After centrifugation, the resultant supernatant was used in subsequent analysis. Total protein concentration was determined with a BCA protein assay method. VEGF concentrations were determined according to the manufacturer's protocols and VEGF levels were expressed as $\mathrm{pg} / \mathrm{mg}$ protein. A minimum of 3 tumors in duplicate per group were analyzed.

2.13. Immunohistochemical analysis of tumor sections: MDA-MB- 231 tumors were fixed in formalin saline and processed for normal histopathological procedures and 5-10 $\mu \mathrm{m}$ sections were made. After deparaffinization and rehydration, sections were processed for immunohistochemical (IHC) protocols as per our established methods (Godugu, Patel et al. 2013, Godugu, Patel et al. 2014). Antigen retrieval for Cleaved caspase 3, ki67 and CD31 was carried out for $10 \mathrm{~min}$ in $0.01 \mathrm{M}$ sodium citrate buffer $(\mathrm{pH} 6)$, heated at $95^{\circ} \mathrm{C}$ in a steam bath followed by cooling for $30 \mathrm{~min}$. The samples were incubated overnight at $4^{\circ} \mathrm{C}$ with Cleaved caspase 3, ki67 and CD31 antibodies or incubated with biotinylated secondary antibody followed by streptavidin. After exposing the peroxidase treated sections to chromagen substrate, brown color was developed at positive sites. After counter staining the sections with hematoxylin, the Cleaved caspase 3, ki67 and CD31 expressions were identified by presence of the brown cytoplasmic staining. Number of positive cells per filed was quantified by counting 10 different fields from each section. Based on the CD31 positive cells, microvessel density (MVD) was calculated. 


\section{Results}

3.1. In vitro anticancer activity of DIM-10 and DIM-14: Both DIM-10 and DIM-14 exhibited concentration dependant increase in the anticancer effects; the cell viability was decreased with increased concentration. The $\mathrm{IC}_{50}$ values were in the range of $10-20 \mu \mathrm{M}$ on different breast cancer cell types. $72 \mathrm{~h}$ after the treatment, the $\mathrm{IC}_{50}$ values of DIM-10 on MDA-MB-231, MDAMB-435, MDA-MB-468, BT474 and SKBR3 cells were found to be $11.63 \pm 0.89,12.45 \pm 1.84$, $11.03 \pm 1.04,13.83 \pm 1.21$ and $8.10 \pm 0.73 \mu \mathrm{M}$, respectively. In case of DIM-14, the $\mathrm{IC}_{50}$ values 72h after the treatment on MDA-MB-231, MDA-MB-435, MDA-MB-468, BT474 and SKBR3 cells were found to be $18.86 \pm 1.62,13.30 \pm 2.46,14.11 \pm 1.30,17.05 \pm 2.24$ and $11.75 \pm$ $1.62 \mu \mathrm{M}$, respectively (Figure $1 \mathrm{~A}-\mathrm{F}$ ). The clonogenic assay also demonstrated the significant anticancer effects of DIM-10 and DIM-14 (Figure 2A). Figure 2D shows representative images of colonies stained with crystal violet. At the highest concentration studied, both DIM-10 and DIM-14 produced 3.39 and 2.26-fold significant reduction in the spheroid number. Further, the spheroid sizes were found to be concentration dependently decreased, at $10 \mu \mathrm{M}$ concentration resulted in 7.66 and 6.17-fold, respectively significant decrease in the spheroid sizes (Figure 3B). The 2D migration gap closure assay performed with C-DIMs also showed significant $(\mathrm{p}<0.001)$ decrease in gap closing compared to control. DIM-10 and DIM-14 showed 2.95 and 2.49-fold, respectively significant decrease in the gap closure, whereas in control cells due to high metastatic migratory nature the gap was narrowed down. Figure $2 \mathrm{C}$ shows the different gap widths calculated in different groups with MDA-MB-231 based cell migration assay. Figure $2 \mathrm{E}$ shows the representative images of cell migration assays.

3.2. Solubility and lipophilicity of DIM-10 and DIM-14: The experimental solubility of DIM-10 was found to be $0.54 \pm 0.06 \mu \mathrm{g} / \mathrm{ml}$ at $\mathrm{pH} 7.4$, whereas the solubility of DIM-14 was found to be $0.31 \pm 0.04 \mu \mathrm{g} / \mathrm{ml}$. The solubility of both these drugs were also not altered at $\mathrm{pH} 2$ and 8 , 
suggesting that the solubility of DIM-10 and -14 was relatively independent of $\mathrm{pH}$ in the range of $\mathrm{pH} 2$ to $8(p>0.05)$.

3.3. Characterization of DIM-10 and DIM-10 NLC formulations: The average sizes of the CDIM derivates loaded NLC particles were found to be in $210-222 \mathrm{~nm}$ range. The zeta potentials were -21.43 and $-14.34 \mathrm{mV}$, respectively for DIM-10 and DIM-14 NLC formulations. The entrapment efficiency of DIM-10 and DIM-14 into the NLC particles were found to be 86.23 and $91.68 \%$, respectively (Table 1$)$.

3.4. Bidirectional permeability of DIM-10 and DIM-14: The TEER values and mean permeability values of paracellular control Lucifer Yellow were $>450 \Omega \cdot \mathrm{cm}^{2}$ and $<0.15 \times 10^{-6}$ $\mathrm{cm} / \mathrm{s}$, respectively. These TEER and permeability values are within normal limits, thus confirms the paracellular integrity of Caco-2 monolayers. The average $P_{\text {eff, }}$ A-B of DIM-10 across Caco-2 cell monolayers was $0.28 \pm 0.05 \times 10^{-6} \mathrm{~cm} / \mathrm{s}$, and $B-A$ was $0.43 \pm 0.06 \times 10^{-6} \mathrm{~cm} / \mathrm{s}$ (Figure $3 A$ ). In case of DIM-14 The average $P_{\text {eff, }}$ A-B of across Caco-2 cell monolayers was $0.26 \pm 0.04 \times 10^{-6}$ $\mathrm{cm} / \mathrm{s}$, and B-A was $0.38 \pm 0.07 \times 10^{-6} \mathrm{~cm} / \mathrm{s}$ (Figure $3 \mathrm{~B}$ ). With both DIM-10 and DIM-14, the Caco-2 permeability studies performed at different $\mathrm{pH}$ conditions $(5,5.8$ and 6.8$)$ did not differ in the permeability (Figure 3A\&B). The TEER values were not significantly changed throughout the Caco-2 permeability studies with DIM-10 and DIM-14, which is indicative of tight junction integrity upon treatment with these drugs. The $P_{\text {eff, }}$ A-B of DIM-10 NLC formulation across Caco-2 cell monolayers was $0.85 \pm 0.02 \times 10^{-5} \mathrm{~cm} / \mathrm{s}$, and for DIM-14 NLC formulation the average $P_{\text {eff }}$, A-B of across Caco- 2 cell monolayers was $0.72 \pm 0.04 \times 10^{-5} \mathrm{~cm} / \mathrm{s}$, respectively. Compared to free drugs, the $\mathrm{P}_{\text {eff }} \mathrm{A}-\mathrm{B}$ of DIM-10 and DIM-14 NLC formulations were found to be 2.59 and 2.56-fold significantly increased (Figure 3C\&D). The in vitro drug release studies indicated that both DIM-10 and DIM-14 NLC formulations showed sustained drug release profile (Figure 3E\&F). The percentage of drug release was significantly increased in NLC formulation groups compared to free drug groups. The maximum \% of drug release found to have increased 
by 2.94 and 3.08 -fold, respectively in DIM-10 and DIM-14 NLC formulations compared to respective free drugs. After $72 \mathrm{~h}$, the cumulative amount of DIM-10 released in free drug and NCL formulation was found to be 30.2 and $88.7 \mu \mathrm{g} / \mathrm{ml}$, respectively. Whereas the cumulative amount of DIM-14 released in free drug and NLC formulations was found to be 29.8 and 91.9 $\mu \mathrm{g} / \mathrm{ml}$, respectively. These released amounts in NLC formulation groups seem to be above the normal solubility limits of these compounds, however, due to increased solubility of DIM-10 and DIM-14 in NLC form might have achieved increased solubility through super saturation. These in vitro drug release studies suggest the increased solubility and sustained release behavior of C-DIM derivates in NLC formulations.

\subsection{Pharmacokinetic analysis of DIM-10, DIM-14 free drugs and NLC Nanoformulations:}

Initial pharmacokinetic studies in rats with DIM-10 and DIM-14 showed limited oral absorption. DIM-10 at 30 and $100 \mathrm{mg} / \mathrm{kg}$ doses, the $\mathrm{C}_{\max }$ concentrations were found to be $2.68 \pm 0.27$ and $8.77 \pm 0.87 \mu \mathrm{g} / \mathrm{ml}$, respectively. At these doses, the AUC levels were found to be $9.56 \pm 0.97$ and 34.42 $\pm 4.12 \mu \mathrm{g} \cdot \mathrm{hr} / \mathrm{ml}$, respectively. In the DIM-10 NLC formulation treated group, significant increase in the oral absorption profile was observed. Compared to DIM-10 free drug, NLC formulation resulted in significant increase in the pharmacokinetic parameters like $C_{\max }, A U C$ and MRT was observed. The $\mathrm{C}_{\max }$ concentration in DIM-10 formulation was 4.73 fold increased. The AUC levels were 11.19 fold significantly increased in NLC formulations compared to DIM10 free drug group (Figure 3G and Table 2). Another drug DIM-14 NLC nanoformulation also exhibited superior oral bioavailability profile compared to free drug. The $\mathrm{C}_{\max }$ and AUC levels were 2.12 and 8.31 fold significantly increased in DIM-14 NLC formulations $(p<0.001)$ compared to free drugs (Figure $3 \mathrm{H}$ and Table 3).

3.6. Pharmacokinetic analysis of DIM-10 NLC in dogs: In dogs, DIM-10 free drug at $5 \mathrm{mg} / \mathrm{kg}$, the $C_{\max }$ levels were found to be $2.5 \pm 0.24 \mu \mathrm{g} / \mathrm{ml}$, whereas in DIM-10 NLC formulations at the same $5 \mathrm{mg} / \mathrm{kg}$ dose, $\mathrm{C}_{\max }$ concentrations were found to be $6.55 \pm 0.64 \mu \mathrm{g} / \mathrm{ml}$. There was a 2.62 
fold increase in the $C_{\max }$ levels in DIM-10 NLC formulations compared to free drug $(p<0.001)$. AUC levels were found to be $42.69 \pm 4.08$ and $125.90 \pm 35.78 \mu \mathrm{g} . \mathrm{hr} / \mathrm{ml}$, respectively in DIM 10 free drug and DIM-10 NLC formulations. The fold increase was found to be 2.94. The percentage oral bioavailability was $27.09 \pm 3.57 \%$ in DIM-10 free drug and in NLC formulation it was $80.06 \pm 8.36 \%$. There is a 2.95 fold significant increase $(p<0.001)$ in the absolute oral bioavailability of DIM-10 in NLC formulation compared to free drug in dogs pharmacokinetic study. The mean residence time (MRT) and $\mathrm{t}_{1 / 2}$ values were also significantly increased in NLC formulations (Figure 3 I and Table 4). In dogs PK study, treatment with DIM 10 NLC formulation at $5 \mathrm{mg} / \mathrm{kg}$ by oral route produced sustained drug concentrations and the plasma concentrations were found to be significantly higher in NLC group ( $3 \mu \mathrm{g} / \mathrm{ml})$ at 24 post dosing. Several factors might have contributed for this effect such as 1) sustained release of drug from NLC particles which are uptaken intact into GIT epithelial cells, 2) altered metabolism of drug in NLC form and 3) decreased hepatic metabolism of drug in NLC form due to lymphatic uptake pathways.

\subsection{Anticancer effects of DIM-10 and DIM-14 NLC formulations in TNBC xenograft}

models: In orthotopic TNBC models, anticancer effects of DIM-10 and DIM-14 free drugs and NLC formulations were evaluated at the dose of $30 \mathrm{mg} / \mathrm{kg}$. Treatment was started 1 week after the tumor cell implantation and continued for 4 weeks. Compared to untreated control groups, DIM-10 free drug and NLC based formulations produced significant anticancer effects in xenograft models. Compared to free drug, DIM-10 NLC formulation produced significantly higher reduction in the tumor volume (Figure 4A\&B). The tumor weights were also significantly reduced in DIM-10 NLC formulations compared to free drug treated animals (Figure 4C). Figure 4D shows the representative tumor bearing animals and isolated tumors in different groups. The anticancer effects of DIM-14 also demonstrated similar kind of anticancer effects like DIM-10. DIM-14 NLC formulations produced significantly higher reduction in the tumor volumes (Figure 5A). DIM-14 formulation produced 2.12 higher anticancer effect compared to free drug treated 
group (Figure 5B). Similarly, DIM-14 oral formulation, 4 weeks after the treatment resulted in 2.56 fold significant reduction $(p<0.001)$ in the tumor weight compared to free drug treated animals (Figure 5C). Figure 5D shows the representative tumor bearing animals and isolated tumors in different groups. The anticancer studies indicate that both DIM-10 and DIM-14 formulations possess superior anticancer effects in vivo in TNBC models.

3.8. Western Blot analysis of DIM-10 and DIM-14 treated tumors samples: The western blot analysis also supported the superior anticancer effects of DIM-10 and DIM-14 in NLC formulations. Compared to control tumors, Cleaved caspase 3 expression was 1.90 fold increased in DIM-10 free drug (FD) treated tumors, whereas in DIM-10-NLC treated tumors its expression was 4.73 fold significantly increased (Figure 6A and D). PNCA expression was 1.19 fold decreased in DIM-10 free drug treated tumors, whereas in DIM-10 NLC formulations, PCNA expression was 2.05 fold decreased (Figure 6A and B). Further, the cyclin D1 expression was 1.12 and 2.05 fold, respectively decreased in DIM-10 free drug and NLC formulation treated tumors compared to untreated control tumors (Figure 6A and C). All the studied markers, DIM10 NLC formulation produced, 2.48, 1.72 and 1.82 fold respective change in cleaved caspase 3 , PCNA and cyclin D1 expression compared to DIM-10 free drug treated tumors. Treatment with DIM-14 also produced favorable anticancer effects. The cleaved caspase 3, which is an apoptotic marker was 1.56 and 2.68 fold, respectively increased in DIM-14 free drug and NLC formulation treated groups (Figure 7A and C). The PNCA expression was 1.28 and 3.51 fold $(p<0.05$ and $p<0.001)$, respectively significantly decreased in free drug and DIM-14 NLC formulation treated groups (Figure 7A and B). Similarly, cyclin D1 expression was 2.28 and 6.63 fold down regulated $(p<0.001)$ in free drug and formulation treated groups (Figure 7A and $D$ ). DIM-14 NLC formulation produced, $1.71,2.74$ and 2.90 fold respective change $(p<0.001)$ in cleaved caspase 3, PCNA and cyclin D1 expression compared to DIM-14 free drug treated tumors (Figure 7). 
3.9. Immunohistochemical analysis of tumors: The IHC analysis of TNBC tumor sections also indicated the superior anticancer effects of DIM-10 and DIM-14 NLC formulations compared to free drug treated tumors (Figure 8A). Cleaved caspase 3 was found to be 2.26 and 2.78 fold significantly increased in DIM-10 free drug and NLC formulation groups (Figure 8B). The increased Cleaved caspase 3 suggest that these compounds produce anticancer effects through induction of apoptosis. Due to anticancer effects of DIM-10, a significant decrease in the cell proliferation marker Ki67 was observed. A 1.42 and 2.90 fold $(p<0.01$ and $p<0.001)$ decrease in the Ki-67 expression was observed in DIM-10 free drug and DIM-10 NLC formulation treated tumors (Figure 8A and C). The CD31 which is indicator of microvascular density (MVD) of tumors was found to be 1.60 and 2.47 fold significantly decreased in DIM-10 free drug and NLC formulation treated tumors compared to control tumors (Figure 8A and D). In all the three markers studied (cleaved caspase 3, Ki-67 and CD31), NLC formulation produced 1.22, 2.04 and 1.54 fold significant change compared to free DIM treated groups (Figure 8A-D). Similar pattern of increased anticancer effects in IHC analysis was observed with DIM-14 NLC formulations (data not shown). The body weight changes and histopathological examination of DIM-10 and DIM-14 as in free drug and NLC formulations did not induce any kind of adverse effects.

3.10. Effect of DIM-10 and DIM-14 on VEGF levels: VEGF levels in tumor lysates were significantly reduced in DIM-10 and DIM-14 free drug and NLC formulation treated groups. Compared to free drug treated groups, NLC formulations produced more reduction in the VEGF levels. Compared to control tumors, in DIM-10 free drug groups, 1.69-fold reduction and in DIM10-NLC formulation groups, 2.88-fold reduction in the VEGF levels were observed (Figure 8E). Similarly, in DIM-14 treated groups, 1.55 and 2.49-fold respectively decrease in free drug and NLC groups was observed. Compared to free drug, 1.70 and 1.60- fold respective reduction in DIM-10 and DIM-14 NLC formulation groups were observed (Figure 8E). 


\section{Discussion}

Developing the anticancer drugs against TNBC is urgently needed, due to rapid increase in the breast cancer patients. There is no selective and efficient drug available to treat TNBC, currently used drugs are associated with severe toxicity concerns (Jamdade, Sethi et al. 2015, Wahba and El-Hadaad 2015). We have chosen two of C-DIM analogs, DIM-10 and DIM-14 and evaluated them in TNBC models. Cytotoxicity assays revealed that both these C-DIM analogs produced concentration dependent cell kill on MDA-MB-231, MDA-MB-468, MDA-MB-453, BT474 and SKBR3 cell types. Our results are in agreement with the previous reports of promising anticancer effects of C-DIMs in different cancer types (Vanderlaag, Su et al. 2008, Vanderlaag, Su et al. 2010, Boakye, Doddapaneni et al. 2013, Goldberg, Draz et al. 2015, Patel, Godugu et al. 2015).

Approximately $30 \%$ of the NCEs suffer with poor oral bioavailability, due to which pharmaceutical companies discontinue further development of many potent NCEs. In our studies, it was observed that both DIM-10 and DIM- 14 have poor aqueous solubility. The absolute oral bioavailabity of both DIM-10 and DIM-14 was found to be approximately $<20 \%$. Because many studies demonstrated the promising role of different nanoformulations in overcoming poor aqueous solubility (Ratnam, Chandraiah et al. 2009, Thanki, Gangwal et al. 2013, Bregoli, Movia et al. 2015, Shakeri and Sahebkar 2015), we chose NLC formulation approach (Liu, Wang et al. 2014, Tran, Ramasamy et al. 2014, Elmowafy, Samy et al. 2015, Qi, Li et al. 2015). Initially, prepared NLC formulation were subjected to in vitro drug release profiles and found that both DIM-10 and DIM-14 release was significantly increased in NLC formulations compared to free drugs; this increased drug release is due to increase solubility of DIMs in nanocarrier systems. Caco-2 permeability studies demonstrated the increased permeability of DIM-10 and DIM-14 NLC formulations. The increased permeability of C-DIMs through Caco-2 monolayers might mainly taken place through intact NLC uptake into cells. Therefore, the 
increased absorption of drug from NLC based formulations is due to increased drug solubility and NLC based increased permeation into monolayers. This increased oral absorption was further supported by pharmacokinetic studies in rats by increased bioavailability of DIM-10 and DIM-14. NLC formulation of DIM-10 exhibited superior oral bioavailabity profile. The $C_{\max }$, AUC levels of DIM-10 NLC formulation at $30 \mathrm{mg} / \mathrm{kg}$ dose was superior to $100 \mathrm{mg} / \mathrm{kg}$ free drug oral formulation. This suggests that NLC based formulations produced greater than 10-fold increase in the oral bioavailability. Therefore, by using NLC based formulation approaches we can reduce the dose to almost $1 / 3^{\text {rd }}$ of the free drug. This observation clearly demonstrates the usefulness of NLC based oral formulation approach to increase bioavailability of poorly soluble drugs. Similarly, the oral pharmacokinetic profile of DIM-14 NLC formulation was also significantly increased. Curcumin is a classical example of a drug which needs high doses for therapeutic benefits because of poor aqueous solubility and poor permeability (Gupta, Patchva et al. 2013, Ahmad, Alkahtani et al. 2015). Several nanoformulation approaches exhibited significant improvement in oral bioavailability of curcumin (Lee, Loo et al. 2014, Palange, Di Mascolo et al. 2014, Ahmad, Alkahtani et al. 2015). In a similar fashion, using NLC formulation based approach, the doses of DIM-10 and DIM-14 can be significantly reduced. In an effort to increase the clinical relevancy, the pharmacokinetic profile of DIM-10 formulation was studied in dogs. The dog pharmacokinetic data also suggested promising effects of NLC formulations. Upon comparison of DIM-10 NLC formulations in rat and dog models, the $C_{\max }$ values were found to be 2.62-fold significantly increased in dog model compared to 4.73 -fold increased observed in rat oral bioavailability studies. Similarly, the AUC values were found to be 2.94 fold inceased in dogs compared to 10.96-fold significant increase in rat models. Though the level of increase was different between dog and rat models, both the animal models demonstrated significant improvement in oral bioavailability compared to free drug. Upon DIM-10 NLC treatment, compared to dogs, rats exhibited higher bioavailability profile. The probable reason for this varied response may be physiological differences between the models and also varied 
uptake and drug disposal mechanisms. Similar types of observations were also reported by us with self emulsified DIM formulations (Ref). Further, the absolute oral bioavailability of DIM-10 was increased from 27 to $80 \%$ in NLC formulations compared to free drug. The possible reasons for increased oral absorption of NLC formulations are increased solubility in nanoparticle form, increased absorption of intact nanoparticles via lymphatic and other GI uptake mechanisms (Beloqui, Solinis et al. 2013, Shete, Selkar et al. 2014). Our studies are in line with published reports indicating the role of NLC formulations to increase oral bioavailability of several drugs. Further, the effect of GIT degradation to limit the oral bioavailabity of DIM compounds was reported in previous studies (McClements, Li et al. 2015). In both rat and dog models, NLC formulations resulted in increase in the MRT. NLC formulations due to their sustained release behavior might have contributed to the increased residence time of the drug (Okonogi and Riangjanapatee 2015). In dogs, $5 \mathrm{mg} / \mathrm{kg}$ dose of DIM-10 in free drug and NLC form was well tolerated. No evidence of toxicity was noticed in dogs treated with DIM-10, therefore, our DIM-10 NLC formulation may be suitable for the clinical use after appropriate trials are done.

The in vitro cytotoxicity and clonogenic assays indicated that both DIM-10 and DIM-14 have very good anticancer effects. The cell migration assay suggested that both these compounds produced significant antimetastatic effects. However, the promising anticancer effects exhibited by DIM-10 and DIM-14 in cell culture based studies did not translate into similar anticancer effects when studied in orthotopic nude mice models. However, our NLC formulations which have shown significant increase in the oral pharmacokinetic profiles, produced superior anticancer effects compared to DIM-10 and DIM-14 free drug treated animals. The NLC based oral formulation produced 2-3 fold increase in the anticancer effects compared to respective free drug treated groups. Among several possible mechanisms of anticancer effects, both DIM-10 and DIM-14 induced anticancer effect through inhibition of VEGF levels. Significant reduction in 
the VEGF levels were observed in tumor lysates in C-DIM treated groups. The role of VEGF in angiogenesis is very well documented in several studies and antiangiogenic effects of C-DIM was also reported in previous studies, which are in agreement with our present findings (Andey, Patel et al. 2013, Godugu, Doddapaneni et al. 2015). Our NLC based oral formulation approach may provide superior anticancer drug delivery strategies for poorly water soluble drugs to improve the pharmacokinetics problems associated with several interesting anticancer agents.

Another mechanism of anticancer effects of DIM-10 and DIM-14 oral formulation is through induction of apoptosis. The western blot analysis demonstrated significant increase of cleaved caspase 3 levels in free drug and NLC formulation treated groups. The apoptosis inducing potential of C-DIMs in different cancer types has been reported earlier (Lei, Abdelrahim et al. 2008). The decreased expression of cyclin D1 in C-DIMs in free drug and NLC formulations suggest that these compounds inhibit the cell proliferation by blocking the cell cycle dependent kinases. Further, the decreased expression of PCNA confirms the role of these drugs to prevent cell proliferation. Previous studies also demonstrated the effect of C-DIM derivatives on cyclin D1 and PNCA expression (York, Abdelrahim et al. 2007, Andey, Patel et al. 2013). In all the in vivo anticancer effects (Tumor volume and tumor weight) and western blot parameters, demonstrated the superior anticancer effects of DIM-10 and DIM-14 formulations compared to respective free drug groups. This is the first study to demonstrate the anticancer effects of DIM10 and DIM-14 on TNBC models. Further, applying the NLC formulation approach and demonstrating the improved pharmacokinetic profile of these formulations in dog models is unique, scalable and translational. Similarly, the Immunohistochemical (IHC) analysis of tumor sections supported the superior anticancer effects of NLC formulations compared to free drug treated animals. The IHC markers such as Ki 67, cleaved caspase 3 and CD31 were significantly different compared to free drug treated groups. Further, the western blotting analysis also resulted in superior anticancer effects of DIM NLC formulations compared to free 
drugs. Upon chronic administration of C-DIM free drugs and NLC formulations did not induce any kind of toxic effects. The Histopathological evaluation of GIT section ileum did not result in any observable morphological toxic effects on GIT. This strongly suggests that NLC formulations are well tolerated upon chronic administration.

\section{Conclusions}

Our in vitro and in vivo studies demonstrate that novel C-DIM analogs (DIM-10 and DIM-14) have potential anticancer effects in TNBC models.. Further, novel NLC based formulations of DIM-10 and DIM-14 resulted in significant increase in the oral bioavailabity and anticancer activity in animal models. The pharmacokinetic studies in dogs also resulted in significant increase in the oral bioavailability of DIM-10 NLC formulation and suggest its translation potential for clinical application. The anticancer effects of DIM-10 and DIM-14 NLC were found to be superior to free drug treated groups. All the molecular parameters such as western blot and IHC assay confirmed the enhanced anticancer effects of NLC formulations compared to free drug treatment. Therefore, our studies clearly demonstrate the beneficial role of DIM-10 and DIM-14 NLC formulations to treat the TNBC. Further, it is also suggested to use NLC based formulation approach to increase oral bioavailability of poorly water soluble drugs from synthetic and photochemical origin.

\section{Conflict of interest}

The authors report no declarations of interest. 


\section{Acknowledgements}

The authors acknowledge the financial assistance of this research from the Department of Defense (DOD) Breast Cancer Program [Grant \# W81XWH-11-1-0211 to M.S]; the National Institute on Minority Health and Health Disparities (NIMHD) P20 program [Grant \# 1P20MD006738-03; to M.S.] and the National Institutes of Health (NIH) SC-1 grant [Grant \# 5SC1CA161676-03 to M.S]. 


\section{Tables}

Table 1: Different formulation characteristics of DIM-10 and DIM-14 NLC formulations

\begin{tabular}{|c|c|c|}
\hline Parameters & DIM-10 NLC & DIM-14 NLC \\
\hline Size (nm) & $214-222$ & 210 \\
\hline Surface Charge (mV) & -21.43 & -14.34 \\
\hline PDI & 0.19 & 0.25 \\
\hline Drug Loading & $10 \%$ & $10 \%$ \\
\hline $\begin{array}{c}\text { Entrapment Efficiency } \\
(\%)\end{array}$ & $86.23 \%$ & $91.68 \%$ \\
\hline
\end{tabular}

Table 2: The Pharmacokinetic parameters of DIM-10 free drug (at 30 and $100 \mathrm{mg} / \mathrm{kg}$ ) and DIM10 NLC (at $30 \mathrm{mg} / \mathrm{kg}$ ) after oral administration. The table also shows the PK profile of i.v. (5 $\mathrm{mg} / \mathrm{kg}$ ) treated groups. Each data point was represented as mean \pm sem $(\mathrm{n}=3-4)$.

\begin{tabular}{|c|c|c|c|c|}
\hline Parameter & $\begin{array}{c}\text { DIM-10 i.v. } 5 \\
\mathrm{mg} / \mathrm{kg}\end{array}$ & $\begin{array}{l}\text { DIM-10 free drug } \\
\qquad 30 \mathrm{mg} / \mathrm{kg}\end{array}$ & $\begin{array}{l}\text { DIM-10 free drug } \\
\qquad 100 \mathrm{mg} / \mathrm{kg}\end{array}$ & $\begin{array}{c}\text { DIM-10 NLC } 30 \\
\mathrm{mg} / \mathrm{kg}\end{array}$ \\
\hline$T_{\max }(h r)$ & 0.00 & $1 \pm 0.12$ & $1 \pm 1.43$ & $2 \pm 0.24$ \\
\hline$C_{\max }(\mu \mathrm{g} / \mathrm{ml})$ & $14.83 \pm 1.48$ & $2.68 \pm 0.27$ & $8.77 \pm 0.87$ & $12.68 \pm 1.27$ \\
\hline$t_{1 / 2}(h r)$ & $0.78 \pm 0.81$ & $4.41 \pm 0.52$ & $5.26 \pm 0.61$ & $13.47 \pm 1.52$ \\
\hline AUC $(\mu \mathrm{g} \cdot \mathrm{hr} / \mathrm{ml})$ & $15.58 \pm 1.67$ & $9.56 \pm 0.97$ & $34.42 \pm 4.12$ & $107.0 \pm 11.23$ \\
\hline AUMC $(\mu \mathrm{g} . \mathrm{hr} / \mathrm{ml})$ & $14.98 \pm 1.55$ & $58.46 \pm 5.93$ & $247.55 \pm 25.87$ & $1469.56 \pm 148.23$ \\
\hline MRT(hr) & $0.96 \pm 0.16$ & $6.11 \pm 0.67$ & $7.19 \pm 0.83$ & $13.73 \pm 1.46$ \\
\hline
\end{tabular}


Table 3: The Pharmacokinetic parameters of DIM-14 free drug (30 mg/kg) and DIM-14 NLC (at $30 \mathrm{mg} / \mathrm{kg}$ ) after oral administration. The table also shows the PK profile of i.v. $(5 \mathrm{mg} / \mathrm{kg})$ treated groups.

\begin{tabular}{|c|c|c|c|}
\hline Parameter & $\begin{array}{c}\text { DIM-14 i.v. } 5 \\
\text { mg/kg }\end{array}$ & $\begin{array}{l}\text { DIM-14 free drug } \\
30 \mathrm{mg} / \mathrm{kg}\end{array}$ & $\begin{array}{c}\text { DIM-14 NLC } 30 \\
\mathrm{mg} / \mathrm{kg}\end{array}$ \\
\hline$T_{\max }(h r)$ & 0.00 & $1 \pm 0.34$ & $2 \pm 0.45$ \\
\hline$C_{\max }(\mu \mathrm{g} / \mathrm{ml})$ & $21.48 \pm 3.59$ & $4.86 \pm 0.67$ & $10.34 \pm 2.04$ \\
\hline $\mathbf{t}_{1 / 2}(\mathbf{h r})$ & $0.68 \pm 0.31$ & $3.83 \pm 0.61$ & $10.78 \pm 1.22$ \\
\hline AUC $(\mu \mathrm{g} \cdot \mathrm{hr} / \mathrm{ml})$ & $17.83 \pm 3.93$ & $10.13 \pm 1.62$ & $84.20 \pm 15.30$ \\
\hline AUMC $(\mu \mathrm{g} . \mathrm{hr} / \mathrm{ml})$ & $18.34 \pm 2.63$ & $61.01 \pm 7.63$ & $1291.08 \pm 383.66$ \\
\hline MRT(hr) & $0.81 \pm 0.27$ & $5.67 \pm 0.45$ & $11.84 \pm 2.16$ \\
\hline
\end{tabular}

Table 4: The Pharmacokinetic parameters of DIM-10 free drug after oral and i.v. administration at $5 \mathrm{mg} / \mathrm{kg}$ dose and DIM-10 NLC formulation ( $5 \mathrm{mg} / \mathrm{kg}$ oral) in dogs model.

\begin{tabular}{|c|c|c|c|}
\hline Parameter & DIM-10 i.v. 5 $\mathbf{~ m g / k g}$ & $\begin{array}{c}\text { DIM-10 free drug 5 } \\
\mathbf{m g} / \mathbf{k g}\end{array}$ & $\begin{array}{c}\text { DIM-10 NLC 5 } \\
\mathbf{m g} / \mathbf{k g}\end{array}$ \\
\hline $\mathbf{T}_{\max }(\mathbf{h r})$ & 0.00 & $2.00 \pm 0.19$ & $1.00 \pm 0.13$ \\
\hline $\mathbf{C}_{\max }(\boldsymbol{\mu g} / \mathbf{m l})$ & $45.61 \pm 4.38$ & $2.50 \pm 0.24$ & $6.55 \pm 0.64$ \\
\hline $\mathbf{t}_{\mathbf{1 / 2}}(\mathbf{h r})$ & $1.11 \pm 0.16$ & $19.61 \pm 1.87$ & $64.90 \pm 6.25$ \\
\hline $\mathbf{A U C}(\boldsymbol{\mu g} \cdot \mathbf{h r} / \mathbf{m l})$ & $31.78 \pm 3.02$ & $42.69 \pm 4.08$ & $125.90 \pm 35.78$ \\
\hline
\end{tabular}




\begin{tabular}{|c|c|c|c|}
\hline MRT(hr) & $1.50 \pm 0.16$ & $24.99 \pm 2.37$ & $92.21 \pm 8.92$ \\
\hline$\%$ Bioavailability & --- & $27.09 \pm 3.57$ & $80.06 \pm 8.36$ \\
\hline
\end{tabular}




\section{Figure Legends}

Figure 1: Cytotoxicity assay. Effect of DIM-10 and DIM-14 on different breast cancer cell lines, A) BT474, B) SKRB3, C) MDA-MB-468, D) MDA-MB-231, E) MDA-MB-453 and F) The $\mathrm{IC}_{50}$ values of DIM-10 and DIM-14 on all these breast cancer cell lines. Each data is represented as mean $\pm \operatorname{sem}(n=6-8)$.

Figure 2: Effect of DIM compounds on clonogenic and cell migration potential. A) Effect of DIM-10 and DIM-14 on total number of spheroids in clonogenic assay studied with MDA-MB468 cells, B) Effect on the spheroid/colony sizes. C) Effect of DIM-10 and DIM-14 on cell migration potential of MDA-MB-231 cells studied by wound/gap closure assay, D) Representative spheroids from different groups and E) Representative images of cell migrations captured at 0 and $48 \mathrm{~h}$ time points. Each data is represented as mean \pm sem $(n=6-8)$. ${ }^{* * *} \mathrm{P}<0.001,{ }^{* *} \mathrm{P}<0.01,{ }^{*} \mathrm{p}<0.05$ Vs control.

Figure 3: Caco-2 permeability study. A and B) Apical to Basolateral permeability of DIM-10 and DIM-14 at different pH conditions, C and D) Caco-2 permeability of DIM-10 and DIM-14 NLC formulations at $\mathrm{pH} 5.8, \mathbf{E}$ and $\mathbf{F}$ ) The in vitro drug release profile of DIM-10 and DIM-14 NLC formulations. Each data point was represented as mean \pm sem $(n=3-4)$. ${ }^{* *} P<0.001$ Vs control group. Oral Pharmacokinetic study of DIM-10 and 14 in Rats, G) Pharmacokinetic profile of DIM-10 free drug (at 30 and $100 \mathrm{mg} / \mathrm{kg}$ ) and DIM-10 NLC (at $30 \mathrm{mg} / \mathrm{kg}$ ), H) Pharmacokinetic profile of DIM-14 free drug and DIM-14 NLC (at $30 \mathrm{mg} / \mathrm{kg}$ ) following oral administration to rats. Each data point was represented as mean $\pm \operatorname{sem}(n=5-6)$. I) Oral Pharmacokinetics of DIM-10 NLC in Dogs. Oral Pharmacokinetic profile of DIM-10 free drug and DIM-10 NLC (at $5 \mathrm{mg} / \mathrm{kg}$ ) in dogs. Each data point was represented as mean \pm sem $(\mathrm{n}=3-4)$.

Figure 4: Anticancer effects of DIM-10 NLC formulation in orthotopic TNBC model. A) Tumor volumes during the 4 weeks of DIM-10 NLC oral administration in TNBC bearing mice, 
B) Final tumor volumes C) Final tumor weights after completion of 4 weeks of DIM-10 free drug and NLC treatment at the dose of $30 \mathrm{mg} / \mathrm{kg}$ dose orally and D) Representative photomicrographs of tumor bearing mice and isolated tumors from different groups. Each data point was represented as mean \pm sem $(n=6-8) .{ }^{*} p<0.05$ and ${ }^{* * *} p<0.001$ Vs untreated groups.

Figure 5: Anticancer effects of DIM-14 NLC formulation in TNBC model. A) Tumor volumes during the 4 weeks of DIM-14 NLC oral administration in TNBC bearing mice, B) Final tumor volumes, C) Final tumor weights after completion of 4 weeks of DIM-14 FD and NLC treatment at the dose of $30 \mathrm{mg} / \mathrm{kg}$ dose. D) Representative photomicrographs of tumor bearing mice and isolated tumors from different groups. Each data point was represented as mean \pm sem $(n=6-8)$. ${ }^{* *} p<0.01$ and ${ }^{* * *} p<0.001$ Vs untreated groups.

Figure 6: Western Blot analysis. A) Effect of DIM-10 NLC on PCNA, Cleaved caspase 3 and Cyclin D1 expression in tumors lysates (representative images). Densitometric quantitative analysis of B) PCNA, C) Cyclin D1 and D) Cleaved caspase 3 expression. Each data point is represented as mean $\pm \operatorname{sem}(n=3-4) .{ }^{* * *} P<0.001,{ }^{*} p<0.05$ Vs control groups.

Figure 7: Western Blot analysis. A) Effect of DIM-14 NLC on expression of PCNA, Cleaved caspase 3 and Cyclin D1 in tumors lysates (representative images). Densitometric quantitative analysis of B) PCNA, C) Cleaved caspase 3 and D) Cyclin D1 expression. Each data point is represented as mean \pm sem $(n=3-4) .{ }^{* * *} P<0.001,{ }^{* *} P<0.01,{ }^{*} p<0.05$ Vs control groups.

Figure 8: Histopathology and Immunohistochemical analysis. A) Representative images of Haemotoxylin and Eosin stained tumor sections (first row) and IHC analysis fields for Cleaved caspase 3 (second row), Ki-67 (third row) and CD-31 (fourth row) markers. The brown color stained fields indicate the positive expression of particular markers. Quantitative analysis of IHC study for B) Cleaved caspase 3, C) Ki-67, D) CD-31 (tumor microvascular density and E) VEGF 
levels in tumors. Each data point was represented as mean \pm sem $(n=3) .{ }^{*} p<0.05,{ }^{* *} p<0.01$ and ${ }^{* * *} p<0.001$ Vs control groups and b Vs DIM-10 free drug (FD) group. 


\section{References}

Ahmad, M. Z., S. A. Alkahtani, S. Akhter, F. J. Ahmad, J. Ahmad, M. S. Akhtar, N. Mohsin and B. A. Abdel-Wahab (2015). "Progress in nanotechnology-based drug carrier in designing of curcumin nanomedicines for cancer therapy: current state-of-the-art." J Drug Target: 1-21.

Andey, T., A. Patel, T. Jackson, S. Safe and M. Singh (2013). "1,1-Bis (3'-indolyl)-1-(psubstitutedphenyl)methane compounds inhibit lung cancer cell and tumor growth in a metastasis model." Eur J Pharm Sci 50(2): 227-241.

Beloqui, A., M. A. Solinis, A. R. Gascon, A. del Pozo-Rodriguez, A. des Rieux and V. Preat (2013). "Mechanism of transport of saquinavir-loaded nanostructured lipid carriers across the intestinal barrier." J Control Release 166(2): 115-123.

Boakye, C. H., R. Doddapaneni, P. P. Shah, A. R. Patel, C. Godugu, S. Safe, S. K. Katiyar and M. Singh (2013). "Chemoprevention of skin cancer with 1,1-Bis (3'-indolyl)-1-(aromatic) methane analog through induction of the orphan nuclear receptor, NR4A2 (Nurr1)." PLoS One 8(8): e69519.

Bregoli, L., D. Movia, J. D. Gavigan-Imedio, J. Lysaght, J. Reynolds and A. Prina-Mello (2015). "Nanomedicine applied to translational oncology: A future perspective on cancer treatment." Nanomedicine 12(1):81-103.

Elmowafy, M., A. Samy, M. A. Raslan, A. Salama, R. A. Said, A. E. Abdelaziz, W. El-Eraky, S. El Awdan and T. Viitala (2015). "Enhancement of Bioavailability and Pharmacodynamic Effects of Thymoquinone Via Nanostructured Lipid Carrier (NLC) Formulation." AAPS PharmSciTech 17(3):663-72.

Godugu, C., R. Doddapaneni, A. R. Patel, R. Singh, R. Mercer and M. Singh (2015). "Novel Gefitinib Formulation with Improved Oral Bioavailability in Treatment of A431 Skin Carcinoma." Pharm Res 33(1):137-54.

Godugu, C., A. R. Patel, U. Desai, T. Andey, A. Sams and M. Singh (2013). "AlgiMatrix based 3D cell culture system as an in-vitro tumor model for anticancer studies." PLoS One 8(1): e53708.

Godugu, C., A. R. Patel, R. Doddapaneni, S. Marepally, T. Jackson and M. Singh (2013). "Inhalation delivery of Telmisartan enhances intratumoral distribution of nanoparticles in lung cancer models." J Control Release 172(1): 86-95.

Godugu, C., A. R. Patel, R. Doddapaneni, J. Somagoni and M. Singh (2014). "Approaches to improve the oral bioavailability and effects of novel anticancer drugs berberine and betulinic acid." PLoS One 9(3): e89919. 
Goldberg, A. A., H. Draz, D. Montes-Grajales, J. Olivero-Verbel, S. H. Safe and J. T. Sanderson (2015). "3,3'-Diindolylmethane (DIM) and its ring-substituted halogenated analogs (ring-DIMs) induce differential mechanisms of survival and death in androgen-dependent and -independent prostate cancer cells." Genes Cancer 6(5-6): 265-280.

Gupta, S. C., S. Patchva and B. B. Aggarwal (2013). "Therapeutic roles of curcumin: lessons learned from clinical trials." AAPS J 15(1): 195-218.

Jamdade, V. S., N. Sethi, N. A. Mundhe, P. Kumar, M. Lahkar and N. Sinha (2015). "Therapeutic targets of triple-negative breast cancer: a review." Br J Pharmacol 172(17): 42284237.

Jamdade, V. S., N. Sethi, N. A. Mundhe, P. Kumar, M. Lahkar and N. Sinha (2015). "Therapeutic Targets of Triple Negative Breast Cancer: A Review." Br J Pharmacol.

Jellinck, P. H., H. L. Makin, D. W. Sepkovic and H. L. Bradlow (1993). "Influence of indole carbinols and growth hormone on the metabolism of 4-androstenedione by rat liver microsomes." J Steroid Biochem Mol Biol 46(6): 791-798.

Lee, W. H., C. Y. Loo, P. M. Young, D. Traini, R. S. Mason and R. Rohanizadeh (2014). "Recent advances in curcumin nanoformulation for cancer therapy." Expert Opin Drug Deliv 11(8): 11831201.

Lei, P., M. Abdelrahim, S. D. Cho, X. Liu and S. Safe (2008). "Structure-dependent activation of endoplasmic reticulum stress-mediated apoptosis in pancreatic cancer by 1,1-bis(3'-indoly)-1-(psubstituted phenyl)methanes." Mol Cancer Ther 7(10): 3363-3372.

Liu, Y., L. Wang, Y. Zhao, M. He, X. Zhang, M. Niu and N. Feng (2014). "Nanostructured lipid carriers versus microemulsions for delivery of the poorly water-soluble drug luteolin." $\underline{\ln t \mathrm{~J}}$ Pharm 476(1-2): 169-177.

McClements, D. J., F. Li and H. Xiao (2015). "The Nutraceutical Bioavailability Classification Scheme: Classifying Nutraceuticals According to Factors Limiting their Oral Bioavailability." Annu Rev Food Sci Technol 6: 299-327.

O'Toole, S. A., J. M. Beith, E. K. Millar, R. West, A. McLean, A. Cazet, A. Swarbrick and S. R. Oakes (2013). "Therapeutic targets in triple negative breast cancer." J Clin Pathol 66(6): 530542.

Okonogi, S. and P. Riangjanapatee (2015). "Physicochemical characterization of lycopeneloaded nanostructured lipid carrier formulations for topical administration." Int J Pharm 478(2): 726-735. 
Palange, A. L., D. Di Mascolo, C. Carallo, A. Gnasso and P. Decuzzi (2014). "Lipid-polymer nanoparticles encapsulating curcumin for modulating the vascular deposition of breast cancer cells." Nanomedicine 10(5): 991-1002.

Patel, A. R., C. Godugu, H. Wilson, S. Safe and M. Singh (2015). "Evaluation of Spray BIO-Max DIM-P in Dogs for Oral Bioavailability and in Nu/nu Mice Bearing Orthotopic/Metastatic Lung Tumor Models for Anticancer Activity." Pharm Res 32(7): 2292-2300.

Patlolla, R. R., M. Chougule, A. R. Patel, T. Jackson, P. N. Tata and M. Singh (2010). "Formulation, characterization and pulmonary deposition of nebulized celecoxib encapsulated nanostructured lipid carriers." J Control Release 144(2): 233-241.

Qi, R., Y. Z. Li, C. Chen, Y. N. Cao, M. M. Yu, L. Xu, B. He, X. Jie, W. W. Shen, Y. N. Wang, M. A. van Dongen, G. Q. Liu, M. M. Banaszak Holl, Q. Zhang and X. Ke (2015). "G5-PEG PAMAM dendrimer incorporating nanostructured lipid carriers enhance oral bioavailability and plasma lipid-lowering effect of probucol." J Control Release 210: 160-168.

Ratnam, D. V., G. Chandraiah, A. K. Meena, P. Ramarao and M. N. Kumar (2009). "The coencapsulated antioxidant nanoparticles of ellagic acid and coenzyme Q10 ameliorates hyperlipidemia in high fat diet fed rats." $\underline{\mathrm{J} N a n o s c i}$ Nanotechnol 9(11): 6741-6746.

Reed, G. A., D. W. Arneson, W. C. Putnam, H. J. Smith, J. C. Gray, D. K. Sullivan, M. S. Mayo, J. A. Crowell and A. Hurwitz (2006). "Single-dose and multiple-dose administration of indole-3carbinol to women: pharmacokinetics based on 3,3'-diindolylmethane." Cancer Epidemiol Biomarkers Prev 15(12): 2477-2481.

Reed, G. A., J. M. Sunega, D. K. Sullivan, J. C. Gray, M. S. Mayo, J. A. Crowell and A. Hurwitz (2008). "Single-dose pharmacokinetics and tolerability of absorption-enhanced 3,3'diindolylmethane in healthy subjects." Cancer Epidemiol Biomarkers Prev 17(10): 2619-2624.

Shakeri, A. and A. Sahebkar (2015). "Nanotechnology: A Successful Approach to Improve Oral Bioavailability of Phytochemicals." Recent Pat Drug Deliv Formul.

Shete, H. K., N. Selkar, G. R. Vanage and V. B. Patravale (2014). "Tamoxifen nanostructured lipid carriers: enhanced in vivo antitumor efficacy with reduced adverse drug effects." $\underline{\operatorname{lnt} \mathrm{J}}$ Pharm 468(1-2): 1-14.

Siddiqui, J. A., A. Singh, M. Chagtoo, N. Singh, M. M. Godbole and B. Chakravarti (2015). "Phytochemicals for breast cancer therapy: current status and future implications." $\underline{\text { Curr Cancer }}$ Drug Targets 15(2): 116-135.

Thanki, K., R. P. Gangwal, A. T. Sangamwar and S. Jain (2013). "Oral delivery of anticancer drugs: challenges and opportunities." J Control Release 170(1): 15-40. 
Tran, T. H., T. Ramasamy, D. H. Truong, H. G. Choi, C. S. Yong and J. O. Kim (2014). "Preparation and characterization of fenofibrate-loaded nanostructured lipid carriers for oral bioavailability enhancement." AAPS PharmSciTech 15(6): 1509-1515.

Vanderlaag, K., Y. Su, A. E. Frankel, R. C. Burghardt, R. Barhoumi, G. Chadalapaka, I. Jutooru and S. Safe (2010). "1,1-Bis(3'-indolyl)-1-(p-substituted phenyl)methanes induce autophagic cell death in estrogen receptor negative breast cancer." BMC Cancer 10: 669.

Vanderlaag, K., Y. Su, A. E. Frankel, H. Grage, R. Smith, 3rd, S. Khan and S. Safe (2008). "1,1Bis(3'-indolyl)-1-(p-substituted phenyl)methanes inhibit proliferation of estrogen receptornegative breast cancer cells by activation of multiple pathways." Breast Cancer Res Treat 109(2): 273-283.

Wahba, H. A. and H. A. El-Hadaad (2015). "Current approaches in treatment of triple-negative breast cancer." Cancer Biol Med 12(2): 106-116.

York, M., M. Abdelrahim, S. Chintharlapalli, S. D. Lucero and S. Safe (2007). "1,1-bis(3'-indolyl)1-(p-substitutedphenyl)methanes induce apoptosis and inhibit renal cell carcinoma growth." $\underline{\text { Clin }}$ Cancer Res 13(22 Pt 1): 6743-6752. 

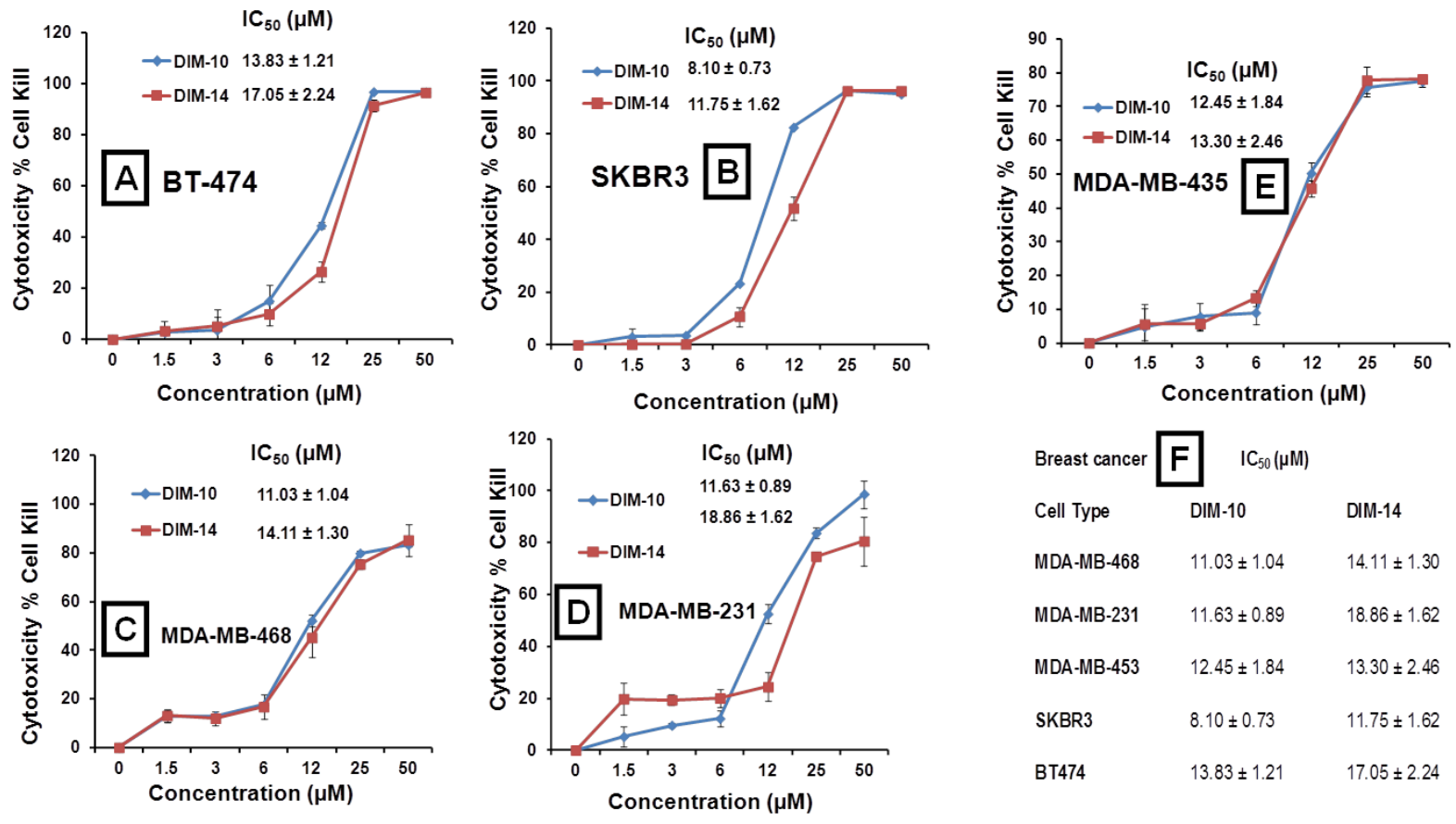

\begin{tabular}{|c|c|c|}
\hline Breast cancer & $\mid \mathrm{IC}_{50}(\mu \mathrm{I}$ & \\
\hline Cell Type & DIM-10 & DIM-14 \\
\hline MDA-MB-468 & $11.03 \pm 1.04$ & $14.11 \pm 1.30$ \\
\hline MDA-MB-231 & $11.63 \pm 0.89$ & $18.86 \pm 1.62$ \\
\hline MDA-MB-453 & $12.45 \pm 1.84$ & $13.30 \pm 2.46$ \\
\hline SKBR3 & $8.10 \pm 0.73$ & $11.75 \pm 1.62$ \\
\hline BT474 & $13.83 \pm 1.21$ & $17.05 \pm 2.24$ \\
\hline
\end{tabular}



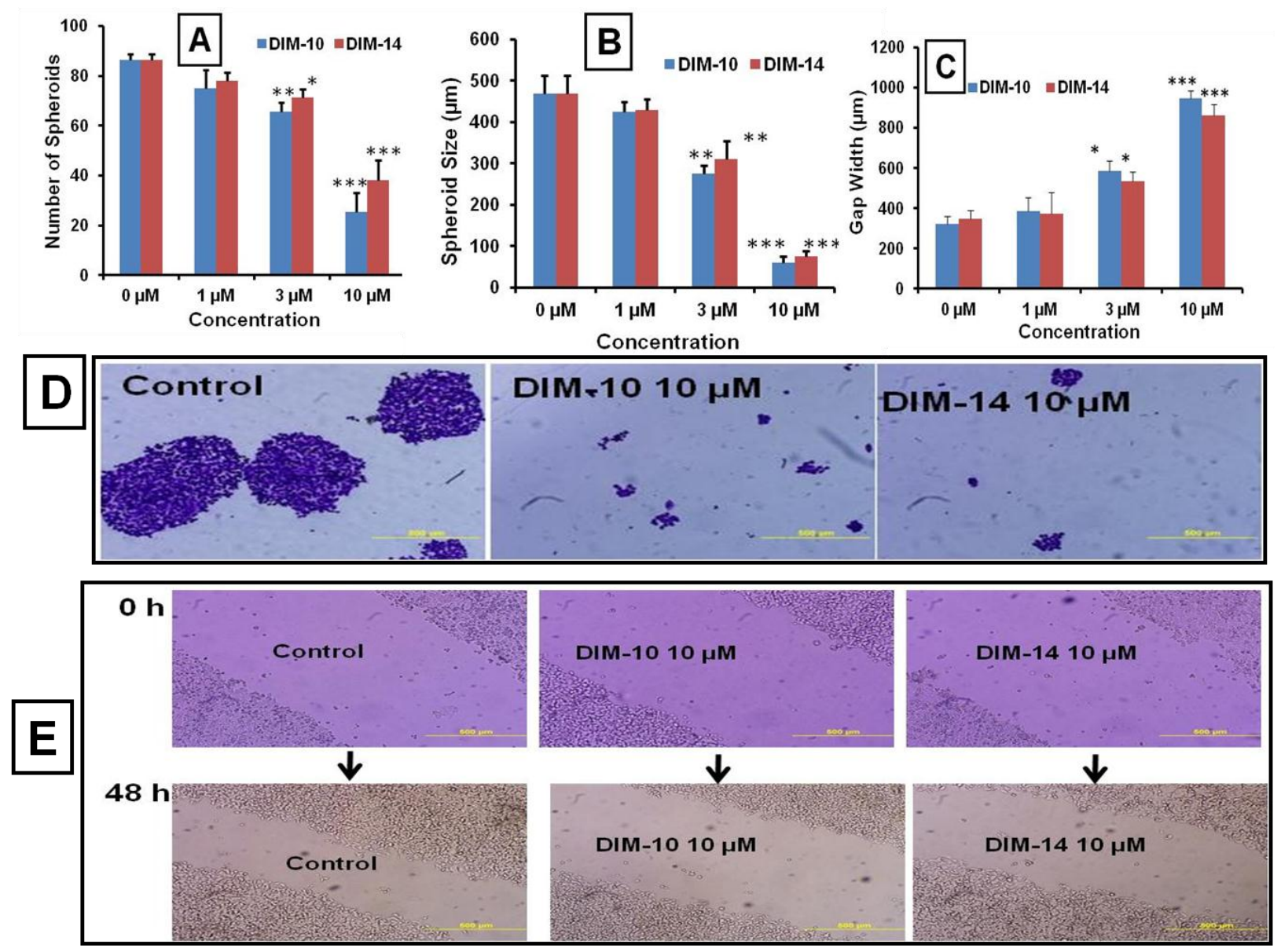


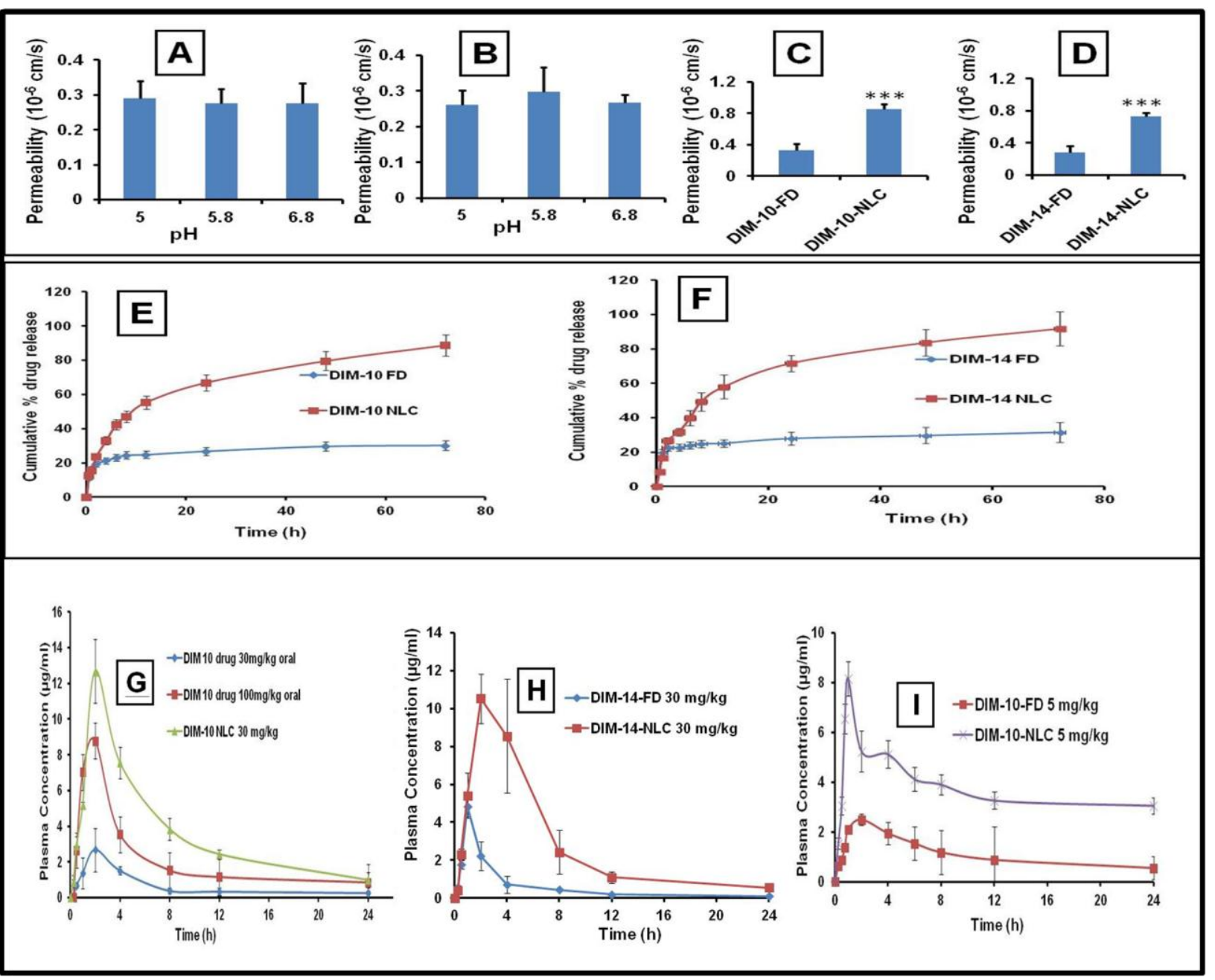



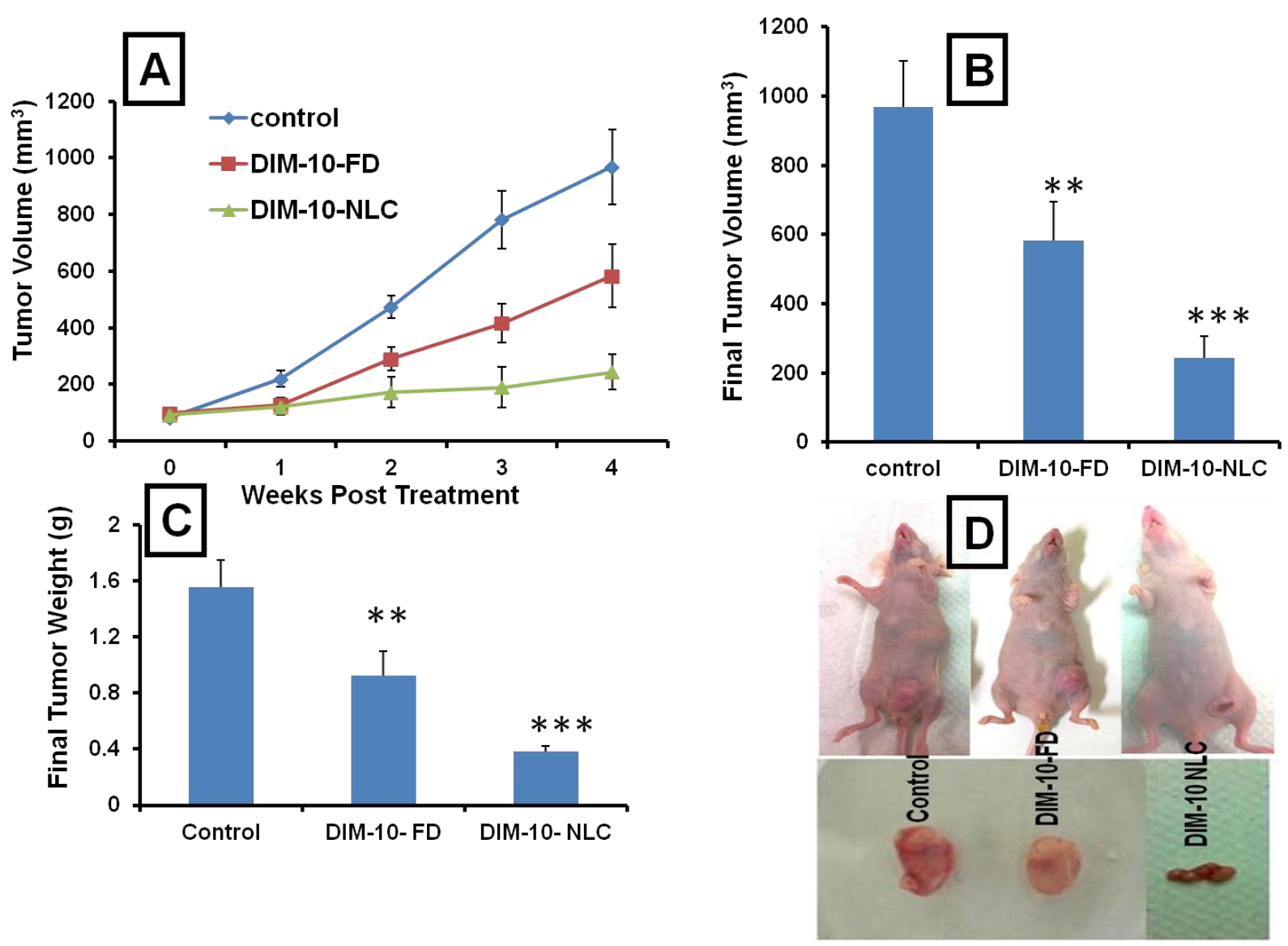

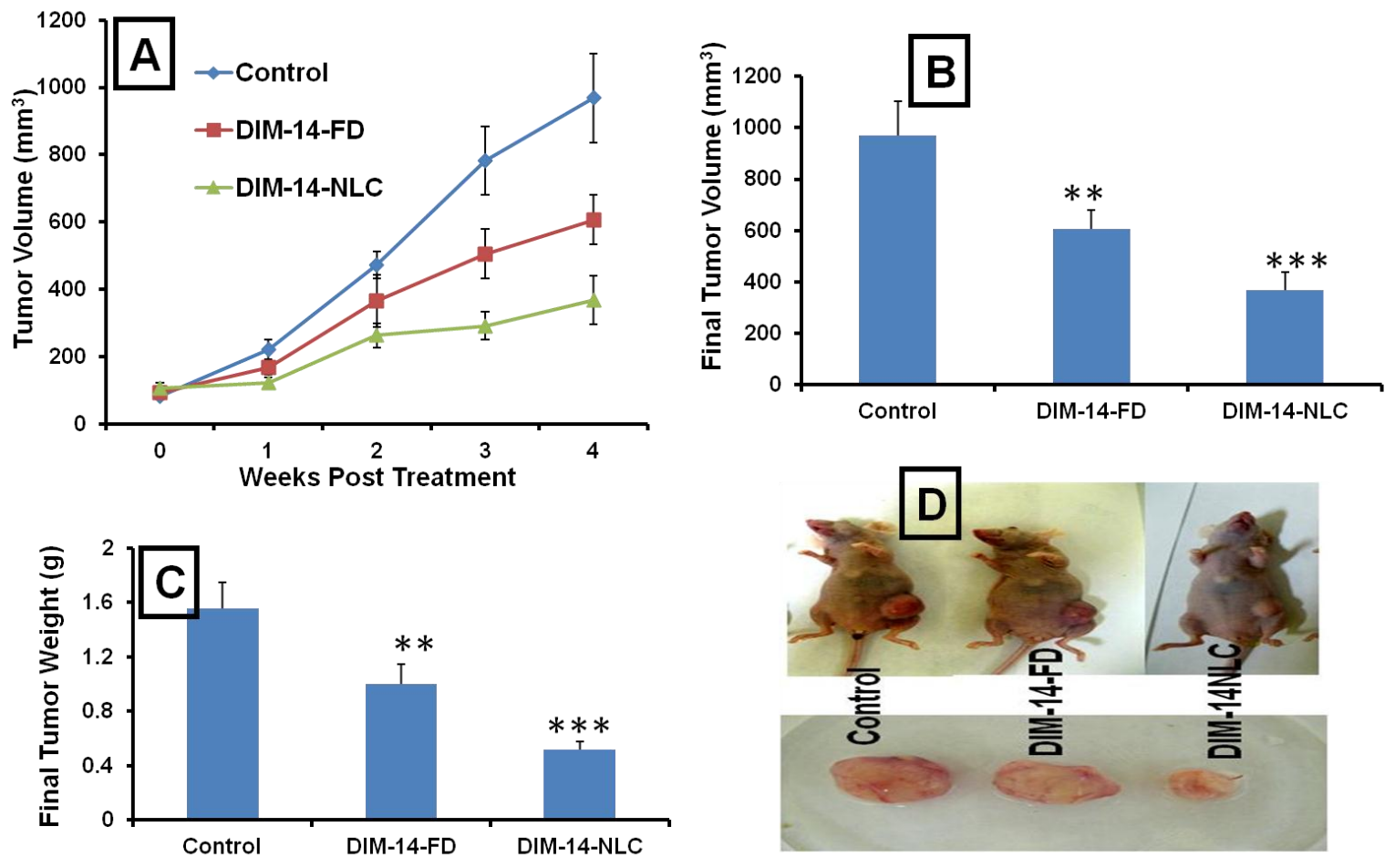

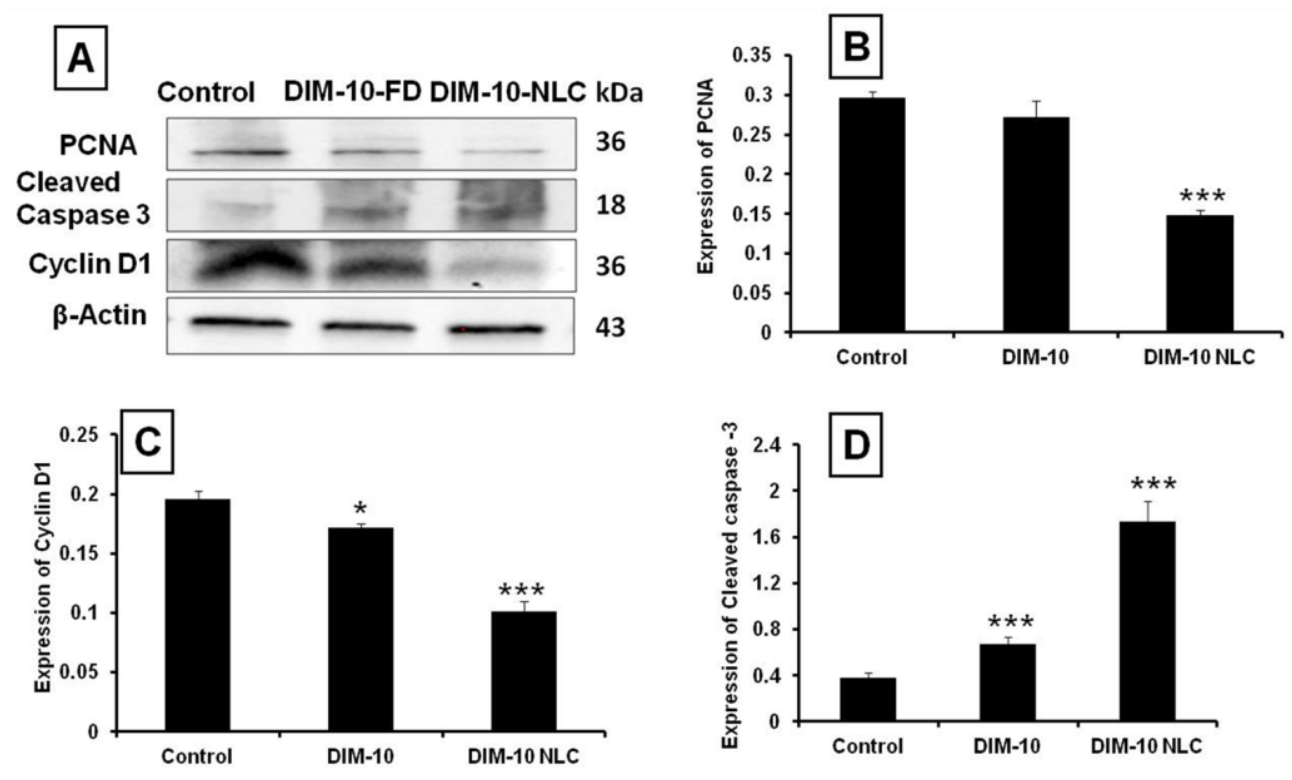

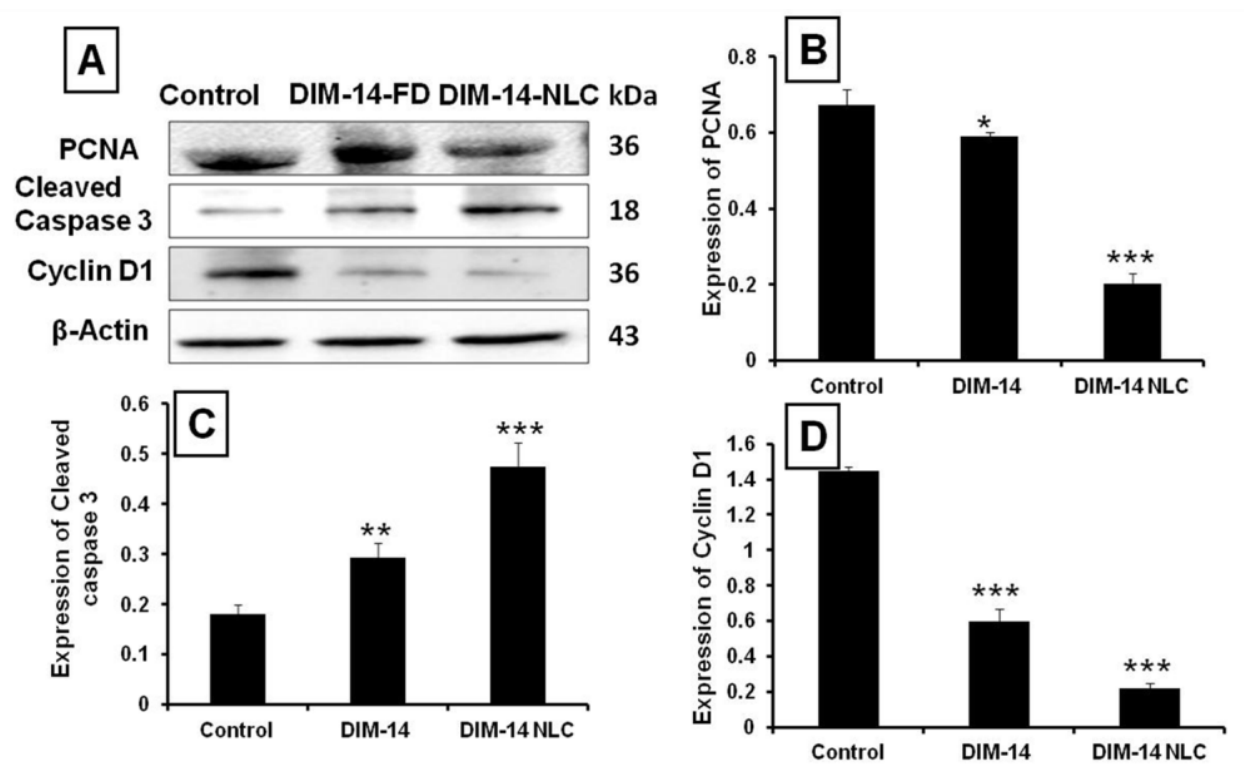


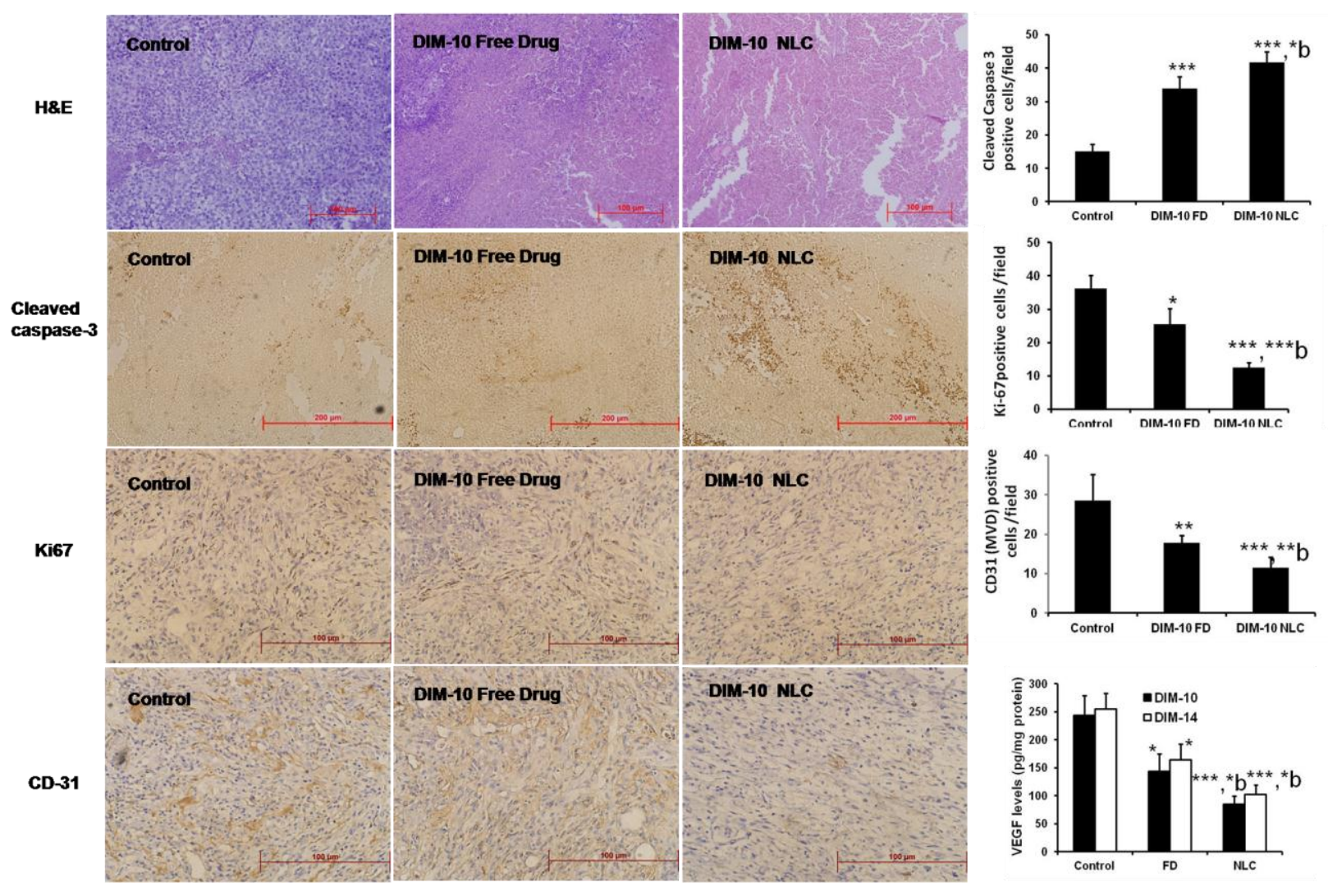




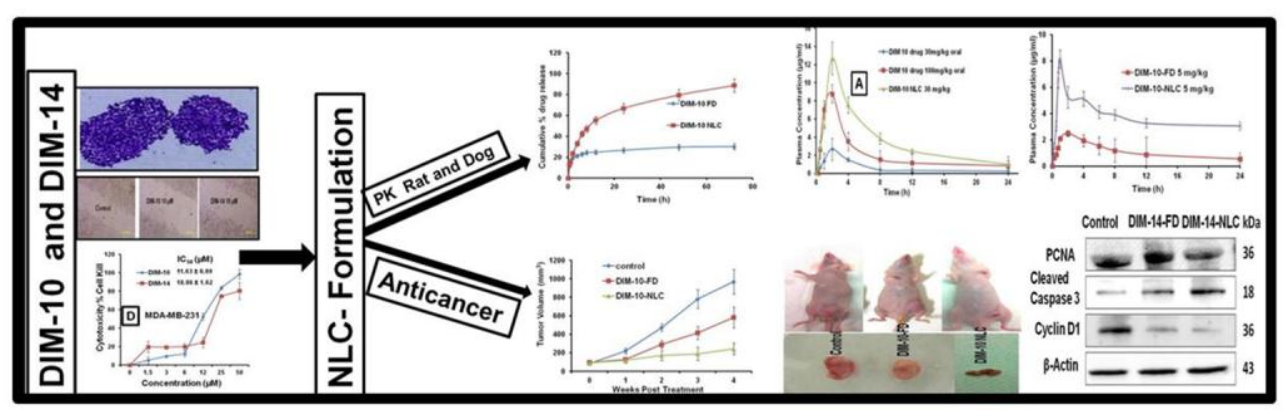

